\title{
Design and Implementation of the Sensor Data Acquisition System Based on Wireless Network Transmission
}

\author{
Boyuan Xu
}

\author{
Academy of Broadcasting Science State Administration of Radio, Film \& Television, China \\ xuboyuan@abs.ac.cn
}

Keywords: Data acquisition with sensor; Wireless data transmission; Compaction degree; Nios II

\begin{abstract}
In the paper put forward a set of scheme that can be suitable for the sensor data acquisition of the compaction degree in the construction of airport runway on the base of the study of the wireless network transmission, sensor data acquisition, digital signal processing and other key techniques, and at last to complete the digital data gathering scheme that will be integrated with the sensor data gathering, wireless network transmission and PC data analyze module. Also the scheme has the advantage of low cost, low power, miniaturization and high integration level, and it could be used in many other situations that need the sensor data acquisition. At last the paper shows the test conclusion in the real environment.
\end{abstract}

\section{Introduction}

Recently, as the development of the wireless communication, integrated circuit and sensor technology, sensor technology based on wireless network transmission is booming rapidly, and digital data acquisition terminal with the sensor being the key part has already been the most important and direct ways for people to obtain the information in different situations. [1][2][3] The main content of this thesis is to put forward a set of scheme that can be suitable for the sensor data acquisition of the compaction degree in the construction of airport runway on the base of the study of the wireless network transmission, sensor data acquisition, digital signal processing and other key techniques, and at last to complete the digital data gathering scheme that will be integrated with the sensor data gathering, wireless network transmission and PC data analyze module.

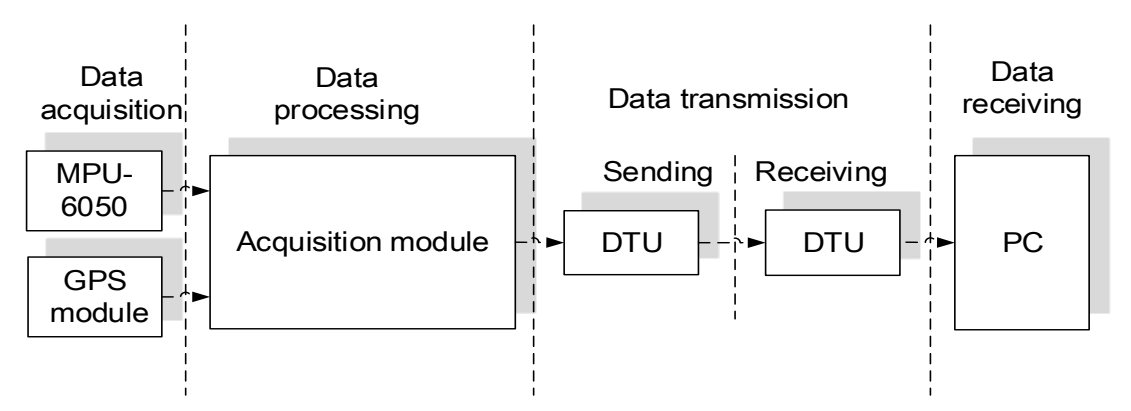

Figure 1. System structure of the design in the paper

In Fig. 1, shows that system in the paper is divided into three parts, including data acquisition part, data transmission part and receiver software. At the data acquisition part, the collection hardware is based on the FPGA board DE0-NANO with the additional circuit. A kind of FHSS data transmission circuit is used for transfer the data from acquisition part to the receiver. The receiver then complete data reception and analysis in Matlab software, that can be provide the reliable compaction degree data in the construction of airport runway for the engineers.

\section{Design of the Acquisition Module}

Fig. 2 shows the system design in the paper with the hardware and software, and it has the features as follows: [4] [5] [6] 


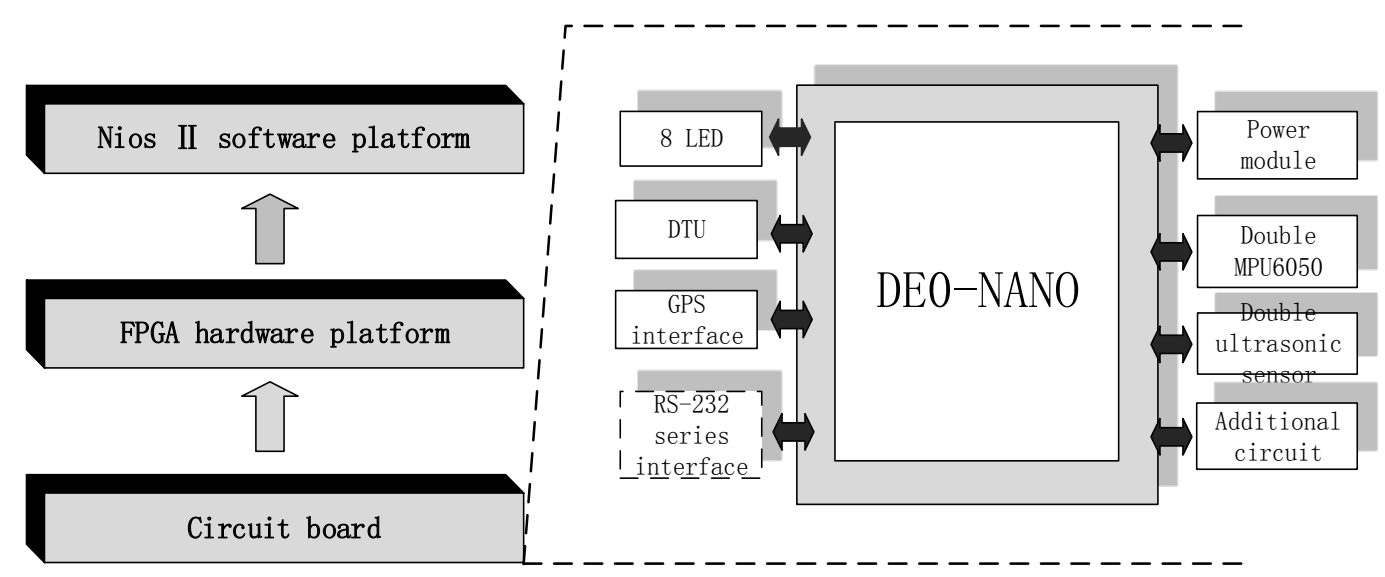

Figure 2. Structure of the hardware and software in acquisition module

(1) Cyclone IV series EP4CE22F17C6N FPGA chip from Altera company is used as the hardware platform, and it has 22320 LEs (Logical Elements), the main running frequency can be $500 \mathrm{MHz}$.

(2) Software development platform is Nios II, development environment is Qsys from Quartus II software.

(3) The base hardware circuit is completed with PCB design, and chip I/O can be connected to the double MPU6050 modules by net wires.

(4) According to the apply circumstance, circuit power should be $12-24 \mathrm{~V} \mathrm{DC}$, and power module can be transfer it to 5V DC for the DE0-NANO and additional circuit.

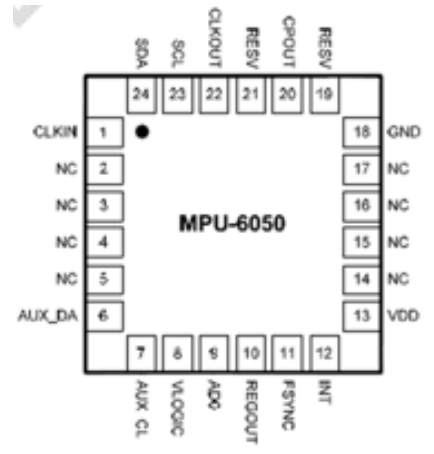

\begin{tabular}{|l|l|}
\hline parameter & feature \\
\hline power & $3-5 \mathrm{~V}$ \\
\hline Communication method & Standard IIC communication protocol \\
\hline AD bits & 16 bit \\
\hline Gyroscope range & $\pm 250 \pm 500 \pm 1000 \pm 2000 \% / \mathrm{s}$ \\
\hline Accelerometer range & $\pm 2 \pm 4 \pm 8 \pm 16 \mathrm{~g}$ \\
\hline
\end{tabular}

Figure 3. Feature of MPU-6050 micro sensor system

The sensor chip adopts the MPU-6050 micro sensor system showed in Fig. 3, and it also shows its electronic parameters. [7] Fig. 4 shows PCB design of the acquisition circuit, and it comprises modules as follows:

(1) MPU-6050 micro sensor system used for data acquisition, with three directions acceleration and angle acceleration data, that can be communication with processing unit through the I2C interface protocol.

(2) The key circuit used for data acquisition has the functions of data reading, filtering and data storage, the main hardware is FPGA chip.

(3) Power circuit module, series interface communication module and other additional circuits. 


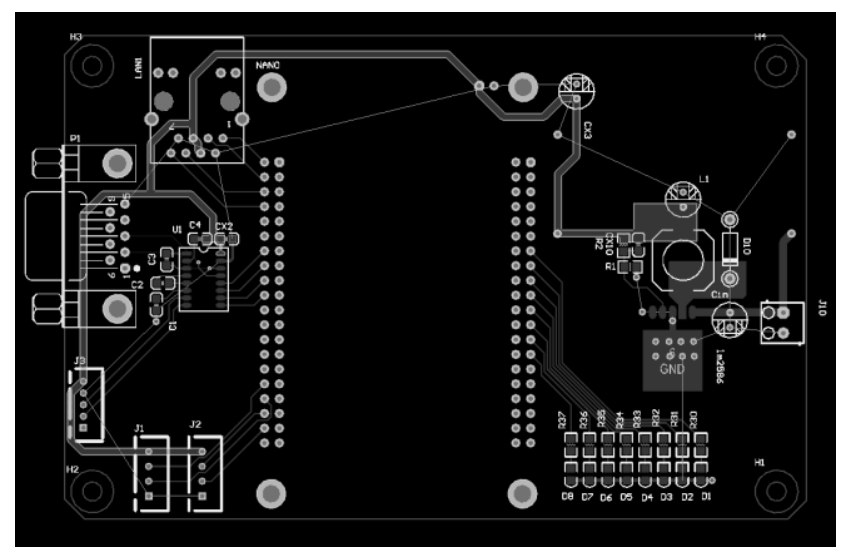

Figure 4. PCB design of the acquisition circuit

\section{Data Transmission Module}

Wireless data transmission is one of the most important parts in the design. According to the apply requirement, data collected from the sensor should be transmit from the acquisition circuit to the PC software receiver by the data transmission module. In the single transmission path, data transmission module need complete the function of sending and receiving in single path, while in the multipath transmission, module should send data from different nodes to the same one point in a polling way. In the design adopts YL-800IL module used for wireless data transmission. YL-800IL has the characteristic of high reliability and strong capacity of resisting disturbance, also it has low power and long transmission distance. YL-800IL has CRC error correction function, and adopts half-duplex communication way. [8] Users can send instructions to the module by the PC software for changing running frequency, signal bandwidth and spreading factor in a flexible way.

\begin{tabular}{|c|c|c|c|c|c|}
\hline Frame head & $\begin{array}{c}\text { Length of } \\
\text { Flag byte }\end{array}$ & $\begin{array}{c}\text { Length of } \\
\text { user data }\end{array}$ & User data & Check bit & End byte \\
\hline $0 \times 55$ & 1 byte & 2 byte & Undefined & 1 byte & $0 \times 1 A$ \\
\hline
\end{tabular}

Figure 5. Structure design of the data frame

In Fig. 5 shows the communication protocol in the design, in which frame head and the last byte is defined with 0x55 and 0xAA in one byte. Different nodes can be defined by the flag byte, such as 0xEA, 0xEB, 0xEC and so on. Then connects 2 bytes used to define user data length, and user data. In the design user data is defined in the application layer protocol, with four bytes used for time stamp, one byte used for packet number and $\mathrm{N}$ data blocks $(\mathrm{N}=50$ in the paper). While data block is defined by the sensor data with 12 bytes.

Table 1 shows the physical layer parameters in the design, and the series interface is configured with one check bit, one stop bit, and baud rate is defined with 9600 .

Table 1 Physical layer parameters in the design

\begin{tabular}{|l|l|}
\hline Parameter & description \\
\hline Baud rate & $9600 \mathrm{Bd}$ \\
\hline Sample frequency & $50 \mathrm{~Hz}$ \\
\hline Numbers of data blocks in one packet & $50 \mathrm{bytes}$ \\
\hline Length of data block & 12 bytes \\
\hline Length of data packet & 605 bytes \\
\hline Length of data frame & 611 bytes \\
\hline
\end{tabular}




\section{Receiver and System Test}

In this design, PC software for the receiver is completed by Matlab software, and it is used for receive and analysis the data from sensor transmitted by the communication module. The analysis data saved in files will be the reliable message for the follow study. Pic shows the work flow of the receiver software.

After the test of sensor feature, system test is completed in the real apply circumstance that is shown in Fig. 6 the impact roller compaction equipment used for building the airport runway. test scheme is that install the sensor on the central axis of the roller compaction equipment, senor should be mounted for steel contact in order to collect the effective data. Sensor connects with the acquisition module with net wire, and acquisition board and wireless communication module is set in the cockpit of the impact roller compaction equipment.

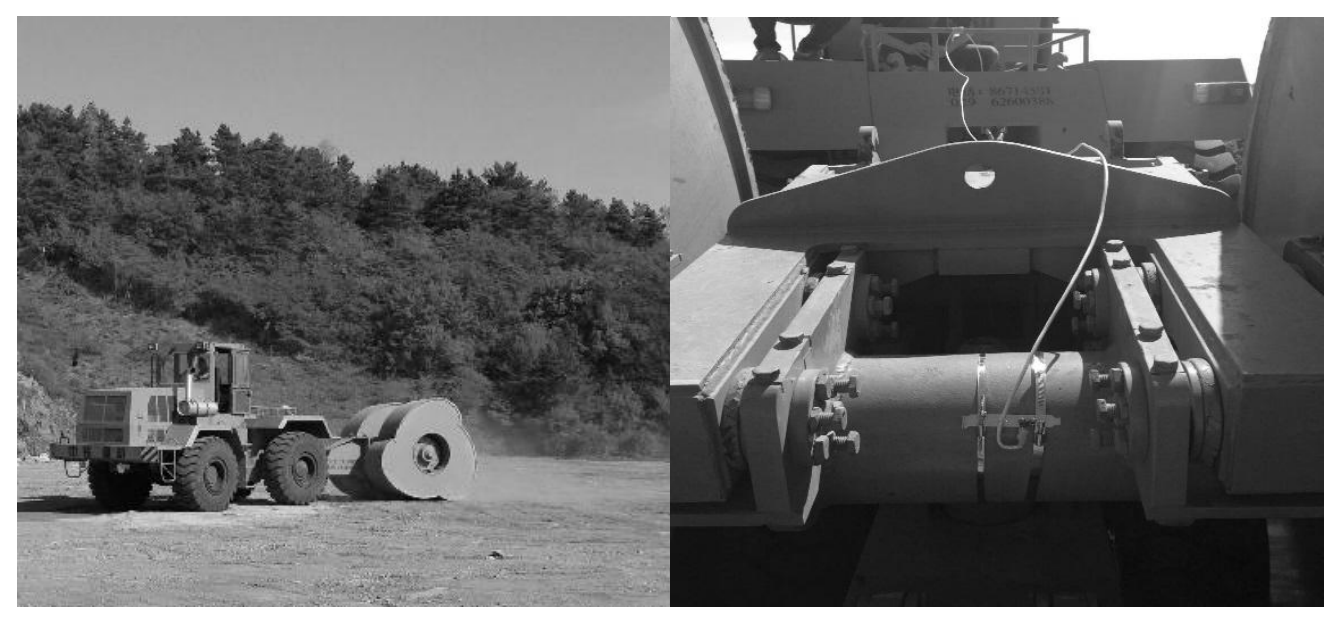

Figure 6. Method of sensor setting on the equipment in real environment

Fig. 7 shows the collected data plot by Matlab software. And it can be seen clearly that once the equipment impacts runway, the acceleration data will accordingly jump, so the sensor data can reflect the impacting processing of the equipment, and the system in the design can be effective to gather data and transmit it to the receiver, then analysis the sensor data for the engineer to compute the compaction degree data.
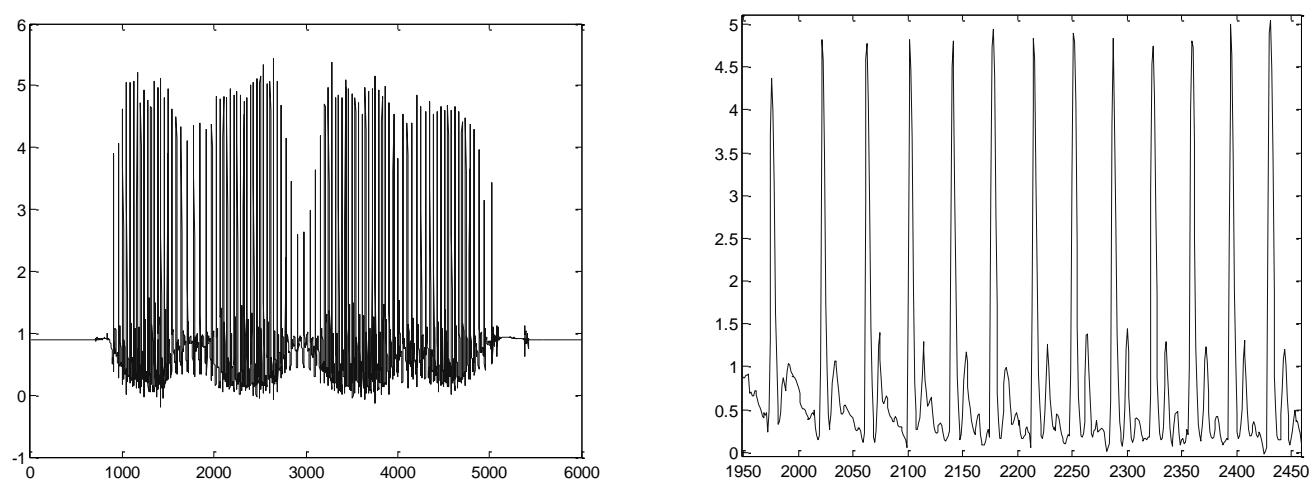

Figure 7. Sensor data from receiver plotted by Matlab software

\section{References}

[1] KRETTEK, OTMAR, ADAMEC, et al. Detecting the Compaction Process by Diagnostics [J]. ZEV-Zeitschrift Fuer Eisenbahnwesen and Verkehrstechnik -Journal for Railway and Transport, 1999, 123(6): 229-236. 
[2] Lars Forssblad, Vibratory Soiland Rock Fill Compaction. Sweden. Stockhol.1981

[3] Chen H, Perrig A, Song D. Random Key Predistribution Schemes for Sensor Networks. IEEE Symposium on Security and Privacy. Berkeley, California, 2003Reference to a book:

[4] Quartus II Handbook, www. Altera. Com.cn

[5] Cyclone IV Device Datasheet(cyiv-53001).pdf

[6] Nios II Software Developer's Handbook

[7] PS-MPU-6000/6500A.pdf

[8] JieXunYiLian-Long distance spread frequency module YL-800IL product sheet.pdf 